\title{
Evaluación de la reproducibilidad de la retinoscopia dinámica monocular de Merchán*
}

\author{
Lizbeth Acuña, Opt, Mag Epidemiol ${ }^{1}$, María Mónica Uribe, Opt, EDU ${ }^{1}$, \\ Luis Carlos Orozco, MD, Mag Epidemiol ${ }^{2}$
}

\section{RESUMEN}

Objetivo: Evaluar la reproducibilidad de la retinoscopia dinámica monocular y su nivel de acuerdo con la retinoscopia estática binocular y monocular, retinoscopia de Nott y Método Estimado Monocular (MEM).

Métodos: Se determinó la reproducibilidad entre los evaluadores y entre los métodos por medio del coeficiente de correlación intraclase (CCI) y se establecieron los límites de acuerdo de Bland y Altman.

Resultados: Se evaluaron 126 personas entre 5 y 39 años y se encontró una baja reproducibilidad interexaminador de la retinoscopia dinámica monocular en ambos ojos CCI ojo derecho: 0.49 (IC95\% 0.36; 0.51); ojo izquierdo 0.51 (IC95\% $0.38 ; 0.59$ ). El límite de acuerdo entre evaluadores fue $\pm 1.25 \mathrm{D}$. Al evaluar la reproducibilidad entre la retinoscopia dinámica monocular y la estática se observó que la mayor reproducibilidad se obtuvo con la estática binocular y monocular y, en visión próxima, entre el método estimado monocular y la retinoscopia de Nott.

Conclusiones: La retinoscopia dinámica monocular no es una prueba reproducible y presenta diferencias clínicas significativas para determinar el estado refractivo, en cuanto a poder dióptrico y tipo de ametropía, por tanto, no se puede considerar dentro de la batería de exámenes aplicados para determinar diagnósticos y correcciones refractivas tanto en la visión lejana como en la visión próxima.

Palabras clave: Retinoscopia dinámica monocular; MEM; Nott; Estática monocular y binocular; Reproducibilidad; Acuerdo.

Evaluation of the reproducibility of the Merchán monocular dynamic retinoscopy

\section{SUMMARY}

Objective: To assess the reproducibility of monocular dynamic retinoscopy and its level of agreement with binocular static retinoscopy, the Nott retinoscopy, and the Monocular Estimated Method (MEM).

Materials and methods: The reproducibility was determined among evaluators and among the methods through the intraclass coefficient of correlation (ICC) and set the limits of agreement of Bland and Altman.

Results: A total of 126 subjects between 5 and 39 years of age were evaluated and low inter-rater reproducibility of the monocular dynamic retinoscopy was found in both eyes: ICC right eye: 0.49 (IC 95\% $0.36 ; 0.61$ ) left eye 0.51 (IC 95\% $0.38 ; 0.59$ ). The limit of agreement between evaluators was $\pm 1.25 \mathrm{D}$. In assessing reproducibility between the monocular dynamic retinoscopy and the static retinoscopy, it was observed that the greatest reproducibility was obtained with the binocular and monocular static retinoscopy and in near vision between the estimated method monocular and Nott retinoscopy.

Conclusions: Monocular dynamic retinoscopy is not a reproducible test and presents clinical differences meaningful to determine the refractive state, regarding power dial and type of ametropy; therefore, it could not be considered within the battery of tests used to determine diagnoses and refractive corrections both in distant vision and in near vision.

Keywords: Monocular dynamic retinoscopy; MEM; Nott; Monocular and binocular static; Reproducibility; Agreement.

* Esta investigación contó con el apoyo de la Universidad Santo Tomás, Bucaramanga, Colombia.

1. Docente, Facultad de Optometría, Universidad Santo Tomas, Bucaramanga, Colombia. e-mail: lizbeth_acuna@yahoo.com.ar mamoniuribe@hotmail.com

2. Docente, Escuela de Enfermería, Universidad Industrial de Santander, Bucaramanga, Colombia. e-mail: lcorovar@uis.edu.co Recibido para publicación junio 17, 2008 Aceptado para publicación septiembre 30, 2009 
La retinoscopia dinámica monocular (RDMM) es una prueba clínica ampliamente utilizada en Colombia propuesta en 1967 por el Dr. Gabriel Merchán de Mendoza para determinar el estado refractivo del ojo, definir el tipo de ametropía y su poder refractivo. Asimismo se usa para el diagnóstico de alteraciones acomodativas que se presentan en el sistema visual en personas con o sin desordenes de la visión binocular ${ }^{1} \mathrm{Su}$ principio se basa en controlar la actividad del cristalino al mantener la fijación sobre un punto localizado en visión próxima ( 40 centímetros), activarla acomodación y eliminar la convergencia, es decir, se realiza monocularmente.

Esta prueba retinoscópica se hace inicialmente con lentes negativos para activar la acomodación y luego se disminyen estos lentes en pasos de 0.25 dioptrías (D) hasta llegar al premovimiento más rápido, es decir un cuarto de dioptría antes de la neutralización del reflejo retinoscópico. El valor dióptrico determinado se denomina dinámico grosso o bruto. Posteriormente se compensa, al valor obtenido en el dinámico groso, $1.25 \mathrm{D}$ negativas en personas de 0 a 40 años de edad que según el autor corresponden a la insuficiencia acomodativa o lag de acomodación propuesta por Bestor (1920) como aparece en Borish ${ }^{2}$, mientras en mayores de 40 años se les compensa el valor de la adición o potencia dióptrica requerida para ver de cerca estimada según la edad.

En el ámbito mundial, la retinoscopia más conocida y aplicada en la clínica para determinar el estado refractivo, es la retinoscopia estática binoculary monocular independiente de los instrumentos que se utilicen para aplicarla. Estudios previamente realizados ${ }^{3}$ demuestran que la reproducibilidad de la retinoscopia estática es superior a 0.80 , y por tanto, se considera como la prueba estándar o de referencia para este propósito.

Respecto a las pruebas para el diagnóstico de alteraciones acomodativas se utilizan la retinoscopia de Nott ${ }^{2}$ y la retinoscopia propuesta por Haynes, Método stimado Monocular (MEM) ${ }^{4}$, las cuales varios autores como Rouse et al. ${ }^{5}$ Locke \& Somers ${ }^{6}$, y Rosenfield \& Portello ${ }^{7}$ compararon y evaluaron la reproducibilidad en ambos métodos obteniendo valores superiores a 0.70 , es decir, buena reproducibilidad, aunque hubo debilidades en los diseños metodológicos de los estudios como tamaños de muestras pequeños, pruebas estadísticas no adecuadas para su análisis, sesgos de selección en la población y no se establece el acuerdo entre pruebas.
Sin embargo, Cacho et al. ${ }^{8}$ y García \& Cacho $^{9}$ evaluaron la reproducibilidad entre Nott y MEM en personas con y sin desordenes de la visión binocular y establecieron el nivel de acuerdo entre ellas, pues vieron que son reproducibles, coeficiente correlación de Pearson $=0.90$, pero sin un buen acuerdo $( \pm 0.53)$ porque presentan gran variabilidad y con diferencias clínicas importantes, es decir superiores a $0.50 \mathrm{D}^{10}$.

No se encuentran estudios publicados que demuestren la calidad de la RDMM o que se compare con las pruebas de referencia y que sea utilizada en otros países (comunicación personal Dr. Bruce Wick, junio 2004). Es decir, esta prueba se utiliza sin tener en cuenta que toda cita diagnóstica se debe aplicar y conocer por lo menos su reproducibilidad ${ }^{11}$, y según Kraemer $^{12}$, para que una prueba sea válida necesariamente debe ser reproducible porque su aplicación es definitiva en las decisiones clínicas, o de lo contrario no es conveniente usarla.

Ante la carencia de evidencia científica, se hizo un estudio de evaluación de pruebas diagnósticas para evaluar la reproducibilidad de la retinoscopia dinámica monocular y su nivel de acuerdo con la retinoscopia estática y la estática monocular. Se comparó con estas dos técnicas: estática binocular porque el autor afirma que la retinoscopia dinámica monocular supera a la retinoscopia estática, pues al controlar la acomodación el resultado es mucho más preciso que al no estarlo y estática monocular porque elimina el efecto de la convergencia y se aplica en otros países.

Igualmente, se evaluó la respuesta acomodativa o poder dióptrico de compensación para hallar el valor dinámico neto en la retinoscopia dinámica monocular de Merchán, con la retinoscopia de Nott y MEM en personas menores de 40 años, a fin de determinar el estado refractivo y el tipo de ametropia en visión lejana debido a que Merchán utiliza en esta población la compensación estándar de $1.25 \mathrm{D}$ sin considerar que la respuesta acomodativa mayor a $1.00 \mathrm{D}$ significa que puede haber alguna deficiencia en el mecanismo de acomodación en este grupo de personas y en mayores de 40 años de edad ${ }^{13,14}$.

\section{MATERIALES Y MÉTODOS}

Se diseñó un estudio de evaluación de tecnología diagnóstica de corte transversal en una muestra de 
hombres y mujeres con edad entre 5 y 39 años, quienes asistieron a la Clínica de Optometría de la Universidad Santo Tomás, Bucaramanga, Colombia, o a través de una búsqueda activa de potenciales participantes en las universidades, colegios del área metropolitana y en sectores de práctica comunitaria donde la Facultad de Optometría presta este servicio y a quienes dos estudiantes de VIII semestre entrenados durante 3 meses, para evitar sesgos, les aplicaron una prueba de selección que consistió en realizar un examen optométrico para determinar si cumplían o no con los criterios de inclusión: personas con edad entre 5 y 39 años, agudeza visual en visión lejana y próxima de 20/20 ó 20/25 sin corrección o con su corrección habitual, flexibilidad de acomodación mayor o igual a seis ciclos por minuto, amplitud de acomodación, aplicando técnica de Donders, hasta 2.00D por debajo de la fórmula de Hofftester $\mathrm{AA}=15-(0.2 *$ edad $)$, reservas de convergencia y divergencia de lejos y cerca según parámetros de normalidad establecidos por Scheiman ${ }^{15}$. Paralelamente se realizó una prueba piloto con 50 participantes para estandarizar los formatos de evaluación y estimar el tamaño de muestra. Se obtuvo un coeficiente de correlación de 0.60 y con estos hallazgos se calculó el tamaño de la muestra, según lo recomendado por Kraemer ${ }^{16}$, con un poder de $80 \%$ y un nivel de significancia de 0.05 que permitiera encontrar un coeficiente de correlación intraclase de 0.6 con una diferencia de 0.15. Estos parámetros determinaron que el tamaño de la muestra debería conformase mínimo con 116. Se evaluaron en total 158 personas de las cuales fueron elegibles 126 participantes.

Se excluyeron del estudio personas con cualquiera de estas condiciones: estrabismo, catarata, afaquia, pseudoafaquia, ambliopía, tratamiento farmacológico ocular usuarios de lentes de contacto y demás alteraciones oculares que impiden o afectan la evaluación del mecanismo acomodativo del ojo y por lo tanto, la refracción. A todos los participantes se les informó sobre los objetivos del estudio y se solicitó su consentimiento por escrito.

Posteriormente, dos optómetras con experiencia clínica y docentes de la Facultad de Optometría de la Universidad Santo Tomás, se encargaron de realizar las pruebas en cada participante aplicando inicialmente la retinoscopia estática binocular y monocular y la retinoscopia dinámica monocular de Merchán. El orden de aplicación de las pruebas fue aleatorizado. Una vez realizadas estas pruebas se aplicó la retinoscopia de Nott y el MEM. Cada prueba se realizó siguiendo los protocolos estándar ${ }^{17}$. Para estos procedimientos se utilizó el retinoscopio de franja $\mathrm{N}^{\circ} 18200$ y una bombilla de luz halógena de 3.5 voltios (Wellch Allyn).

Los dos examinadores se codificaron y fueron enmascarados, ninguno conocía los resultados de las pruebas obtenidas por el otro examinador con el fin de evitar sesgos del diagnóstico. Se registró el valor dióptrico del dinámico grosso o poder refractivo para visión cercana en la retinoscopia dinámica monocular y luego se registró el poder de visión lejana compensando 1.25D para comparar este poder con el obtenido con la retinoscopia estática y estática monocular. Al realizar la retinoscopia de Nott se registró la distancia de neutralización en centímetros y luego se hizo la conversión en dioptrías determinando el valor dinámico grosso tanto en Nott como en MEM para compararlo con el valor de obtenido con la RDMM.

Se realizó un análisis univariado para describir cada una de las variables del estudio. Se estimaron las medidas de tendencia central de las variables continuas y las proporciones de las variables discretas. Se aplicóla prueba de Shapiro Wilk para evaluar la distribución de las variables continuas. Se determinó el equivalente esférico en defectos astigmáticos con el fin de comparar el poder esférico. Para establecer si existían diferencias estadísticamente significativas entre los valores dióptricos obtenidos por cada prueba y cada evaluador se utilizó la prueba de Wilcoxon y la prueba t para datos pareados. Se evaluó la correlación entre las mediciones y finalmente, la reproducibilidad entre los evaluadores, en el ojo derecho e izquierdo, por medio del coeficiente de correlación intraclase y sus intervalos de confianza del $95 \%$. Elnivel de acuerdo entre los evaluadores se determinó con los límites de acuerdo del $95 \%$ de Bland y Altman.

Para el análisis se crearon bases de datos dobles en Epi Info (6.2) ${ }^{18}$ que fueron exportadas al programa STATA $9.0^{19}$ en el cual se realizó el procesamiento y análisis de datos.

Este estudio se consideró de riesgo mínimo, según el Decreto $N^{\circ} 08430$ de 1993 de Colombia y fue aprobado por el Comité de Ética de la Universidad Santo Tomás de Colombia. 
Cuadro 1

Distribución de los valores dióptricos en miopes según cada prueba retinoscópica y cada evaluador

\begin{tabular}{|c|c|c|c|c|}
\hline \multirow{3}{*}{ Restinoscopia } & \multicolumn{4}{|c|}{ MIOPES } \\
\hline & \multicolumn{2}{|c|}{ Evaluador 1 Mediana (RI) ${ }^{1}$} & \multicolumn{2}{|c|}{ Evaluador 2 Mediana (RI) } \\
\hline & OD & OI & OD & OI \\
\hline Estática & $-0.25(\mathrm{RI}:-0.25-0.75)$ & $-0.25(\mathrm{Rl}:-0.25-0.50)$ & $-0.25(\mathrm{Rl}:-0.25-0.50)$ & $-0.25(\mathrm{Rl}:-0.25-0.75)$ \\
\hline $\begin{array}{l}\text { Estática } \\
\text { monocular }\end{array}$ & $-0.50(\mathrm{RI}:-0.25-0.50)$ & $-0.50(\mathrm{Rl}:-0.25-0.50)$ & $-0.50(\mathrm{Rl}:-0.25-0.50)$ & $-0.50(\mathrm{Rl}:-0.25-0.50)$ \\
\hline $\begin{array}{l}\text { Dinámica monocular } \\
\text { de Merchán } \\
\text { (Dinámico neto) }\end{array}$ & $-0.50(\mathrm{Rl}:-0.25-0.75)$ & $-0.50(\mathrm{RI}:-0.2-0.50)$ & $-0.50(\mathrm{Rl}:-0.25-0.50)$ & $-0.50(\mathrm{Rl}:-0.25-0.50)$ \\
\hline
\end{tabular}

1. Rango intercuartílico

Cuadro 2

Distribución de los valores dióptricos en hipermétropes según cada prueba retinoscópica y cada evaluador

\begin{tabular}{|c|c|c|c|c|}
\hline \multirow{3}{*}{ Restinoscopia } & \multicolumn{4}{|c|}{ HIPERMÉTROPES } \\
\hline & \multicolumn{2}{|c|}{ Evaluador 1 Mediana (RI) ${ }^{1}$} & \multicolumn{2}{|c|}{ Evaluador 2 Mediana (RI) } \\
\hline & OD & ol & OD & ol \\
\hline Estática & $0.50(\mathrm{RI}: 0.25-0.75)$ & $0.50(\mathrm{Rl}: 0.25-0.75)$ & $0.50(\mathrm{RI}: 0.25-0.75)$ & $0.50(\mathrm{Rl}: 0.75-0.25)$ \\
\hline $\begin{array}{l}\text { Estática } \\
\text { monocular }\end{array}$ & $0.50(\mathrm{RI}: 0.25-0.75)$ & $0.50(\mathrm{RI}: 0.25-0.75)$ & $0.50(\mathrm{RI}: 0.25-0.75)$ & $0.50(\mathrm{RI}: 0.25-0.75)$ \\
\hline $\begin{array}{l}\text { Dinámica monocular } \\
\text { de Merchán } \\
\text { (Dinámico neto) }\end{array}$ & 0.62 (RI:0.25-0.75) & $0.75(\mathrm{RI}: 0.25-1.0)$ & $0.75(\mathrm{RI}: 0.5-1.0)$ & $0.75(\mathrm{RI}: 0.50-1.0)$ \\
\hline
\end{tabular}

1. Rango intercuartílico

\section{RESULTADOS}

La población estudio estaba constituida por 126 personas con edades entre 5 y 39 años, la mediana fue 13.5 años (rango intercuartílico $\mathrm{RI}=10$ a 20 años). La mayor proporción de los participantes estaba entre $10 \mathrm{y}$ 14 años y sólo $4.7 \%$ entre 35 y 39 años. De los participantes 69 (54.7) eran de sexo femenino. Apenas $7.2 \%$ de los integrantes de la encuesta, usaban anteojos para corregir su alteración refractiva.

En cuanto a los valores dióptricos determinados por cada evaluador en visión lejana, en cada una de las pruebas, no hubo diferencias clínicas ni estadísticamente significativas $\mathrm{p}=0.17$, las diferencias no eran superiores a $0.50 \mathrm{D}$ (Cuadros 1 y 2).

En la reproducibilidad entre examinadores, en las pruebas para visión lejana, se observó que en la retinoscopia estática el coeficiente de correlación fue excelente en ambos ojos, 0.89 (IC 95\% 0.85; 0.92) ojo derecho (OD) y 0.87 (IC 95\% 0.83; 0.91 ) ojo izquierdo (OI) (Cuadro 3). Similares resultados se presentaron en la retinoscopia estática monocular, mientras en la diná- 


\section{Cuadro 3}

Reproducibilidad entre-evaluadores en cada prueba, ojo derecho e izquierdo

\begin{tabular}{lcc}
\hline \multicolumn{1}{c}{ Prueba } & $\begin{array}{c}\text { Ojo derecho E1-E2 } \\
\text { CCI }^{1} \text { (IC95\%) }\end{array}$ & $\begin{array}{c}\text { Ojo izquierdo E1-E2 } \\
\text { CCI (IC95\%) }\end{array}$ \\
\hline Estática & $0.89(0.85 ; 0.92)$ & $0.87(0.83 ; 0.91)$ \\
Estática monocular & $0.82(0.76 ; 0.87)$ & $0.80(0.72 ; 0.85)$ \\
Monocularde Merchán & $0.49(0.36 ; 0.61)$ & $0.51(0.38 ; 0.59)$ \\
MEM & $0.69(0.60 ; 0.78)$ & $0.73(0.65 ; 0.71)$ \\
Nott & $0.81(0.75 ; 0.87)$ & $0.83(0.78 ; 0.88)$ \\
\hline
\end{tabular}

1. E1: Evaluador 1. E2: Evaluador 2

2. CCl: Coeficiente de correlación intraclase (IC95\%); intervalo de confianza de 95\%

\section{Cuadro 4 \\ Límites de acuerdo de $95 \%$ de Bland y Altman entre los evaluadores en cada prueba, ojo derecho e izquierdo}

\begin{tabular}{lcccc}
\hline Prueba & \multicolumn{2}{c}{$\begin{array}{c}\text { Ojo derecho } \\
\text { (Límites de acuerdo de 95\%) }\end{array}$} & $\begin{array}{c}\text { Ojo izquierdo } \\
\text { DE }\end{array}$ & (Límites deacuerdo de 95\%) \\
\hline Estática binocular & 0.29 & $(-0.61 ; 0.52)$ & 0.32 & $(-0.87 ; 0.91)$ \\
Estática monocular & 0.38 & $(-0.75 ; 0.75)$ & 0.40 & $(-0.94 ; 0.82)$ \\
Dinámica monocular & 0.71 & $(-1.47 ; 1.29)$ & 0.70 & $(-1.48 ; 1.26)$ \\
Nott & 0.16 & $(-0.28 ; 0.37)$ & 0.12 & $(-0.21 ; 0.28)$ \\
MEM & 0.16 & $(-0.29 ; 0.36)$ & 0.17 & $(-0.31 ; 0.37)$ \\
\hline
\end{tabular}

1. DE: Desviación estándar de las diferencias entre evaluadores

mica de Merchán la reproducibilidad del coeficiente fue bajo entre 0.49 en el ojo derecho y 0.51 en el izquierdo.

En las pruebas para determinar la respuesta acomodativa, la retinoscopia de Nott presentó buena reproducibilidad, comparada con el MEM(Nott CCI = 0.81 OD y 0.83 OI MEM 0.69 OD y 0.73 OI)

Respecto a los límites de acuerdo entre los evaluadores se encontró que en la retinoscopia estática fue de $\pm 0.50 \mathrm{D}$, la estática monocular $\pm 0.75 \mathrm{D}$, y la retinoscopia dinámica monocular \pm 1.25 D. En la retinoscopia de Nott y MEM se obtuvo un nivel de acuerdo de $\pm 0.25 \mathrm{D}$ (Cuadro 4).

Al evaluar la reproducibilidad entre las pruebas en visión lejana se observó que la mayor reproducibilidad se obtuvo con la estática bino y monocular CCI 0.95 ojo derecho y $0.90 \mathrm{D}$ en el ojo izquierdo (Cuadro 5). El acuerdo entre estas pruebas fue $\pm 0.37 \mathrm{D}$. Al comparar estas dos retinoscopias con la dinámica monocular hubo reproducibilidad promedio de 0.51 y 0.46 respectivamente, con un rango de acuerdo de $95 \%$ de $\pm 1.00 \mathrm{D}$ (Cuadro 5).

En visión próxima la reproducibilidad entre MEM y Nott fue 0.94 en ambos ojos y se obtuvo un acuerdo entre pruebas de $\pm 0.25 \mathrm{D}$.

\section{DISCUSIÓN}

Los hallazgos de esta investigación, demuestran con evidencia científica baja reproducibilidad y acuerdo de la retinoscopia dinámica monocular de Merchán. La población de estudio estuvo conformada por 126 personas entre 5 y 39 años de edad; sin embargo existió baja inclusión de sujetos entre 34 y 39 años debido a que presentaron alteraciones de la acomodación posible- 


\section{Cuadro 5}

Reproducibilidad entre las pruebas retinoscópicas para determinar el poder dióptrico en visión lejana y próxima ojo derecho e izquierdo

\begin{tabular}{lll}
\hline \multicolumn{1}{c}{ Prueba } & $\begin{array}{l}\text { Ojo derecho } \\
\mathbf{C C I}^{1} \text { (IC95\%) }\end{array}$ & $\begin{array}{l}\text { Ojo izquierdo } \\
\text { CCI (IC95\%) }\end{array}$ \\
\hline MEM vs. Nott & $0.94(0.93 ; 0.96)$ & $0.94(0.92 ; 0.96)$ \\
MEM vs. dinámica monocular de Merchán & $0.33(0.24 ; 0.42)$ & $0.22(0.13 ; 0.32)$ \\
Nott vs. dinámica monocular de Merchán & $0.34(0.26 ; 0.43)$ & $0.21(0.12 ; 0.31)$ \\
Estática binocular vs estática monocular & $0.95(0.93 ; 0.97)$ & $0.90(0.87 ; 0.93)$ \\
Estática vs. dinámica monocular de Merchán & $0.61(0.50 ; 0.71)$ & $0.42(0.28 ; 0.56)$ \\
Estática monocular vs dinámica monocular de Merchán & $0.58(0.47 ; 0.69)$ & $0.34(0.19 ; 0.49)$ \\
\hline
\end{tabular}

\section{Cuadro 6}

Límites de acuerdo del 95\% de Bland y Altman entre pruebas, ojo derecho e izquierdo

\begin{tabular}{|c|c|c|c|c|}
\hline & \multicolumn{2}{|l|}{ Ojo derecho } & \multicolumn{2}{|c|}{ Ojo izquierdo } \\
\hline & $\mathrm{DE}^{*}$ (Dioptrías) & $\begin{array}{l}\text { Límites } \mathrm{de} \\
\text { acuerdo del } 95 \%\end{array}$ & DE (Dioptrías) & $\begin{array}{c}\text { Límites de } \\
\text { acuerdo del } 95 \%\end{array}$ \\
\hline Estática vs. estática monocular & 0.18 & $(-0.34 ; 0.37)$ & 0.28 & $(-0.55 ; 0.55)$ \\
\hline Estática vs. dinámica monocular & 0.51 & $(-0.84 ; 1.18)$ & 0.45 & $(-1.45 ; 1.10)$ \\
\hline $\begin{array}{l}\text { Estática monocular vs. dinámica } \\
\text { monocular }\end{array}$ & 0.54 & $(-1.24 ; 0.87)$ & 0.54 & $(-1.24 ; 0.87)$ \\
\hline Nott vs. dinámica monocular & 0.52 & $(-1.8 ; 0.24)$ & 0.70 & $(-2.15 ; 0.59)$ \\
\hline MEM vs. dinámica monocular & 0.53 & $(-1.92 ; 0.24)$ & 0.68 & $(-2.09 ; 0.59)$ \\
\hline Mem vs. Nott & 0.19 & $(-0.38 ; 0.38)$ & 0.20 & $(-0.38 ; 0.43)$ \\
\hline
\end{tabular}

* Desviación estándar del promedio de las diferencias

mente relacionadas con la edad. Asimismo, se obtuvo mayor participación del sexo femenino y no hay evidencia que sugiera diferencias significativas de valores dióptricos por género ${ }^{20}$.

El estado refractivo más frecuente fue la hipermetropía y concuerda con lo expuesto por Grosvenor quien afirma que es la alteración más frecuente en la población ${ }^{21}$ (Cuadro 6).

El poder dióptrico de la respuesta acomodativa o lag de acomodación valorado con MEM y Nott se presentó frecuentemente con valores de 0,50 y $0,75 \mathrm{D}$. Los valores encontrados en esta investigación concuerdan con los hallazgos de García et al. ${ }^{9}$ Sin embargo, en este estudio se evidenció que no hay una relación directa entre la respuesta acomodativa y la edad y que ésta no es una constante, por lo cual se debe evaluar en cada persona para determinar si existe o no alteraciones acomodativas con mayor precisión (Gráfica 1).

Se identificó que una proporción de la población estudio inferior al $2 \%$ presentaron el lag de acomodación con valor de 1,25D. Esto contradice la afirmación de Merchán quien compensa en la refracción dinámica monocular $1,25 \mathrm{D}$, asumiendo que la respuesta acomodativa se presenta de forma constante en la población y por otro lado, este valor en personas jóvenes, según la literatura ${ }^{13}$, corresponde a una disfunción de la acomodación e indica insuficiencia, paresia, hipermetropía latente, y en ocasiones, disfunción de las vergencias mientras que en personas mayores de 40 años es una insuficiencia acomodativa ${ }^{14}$. Asimismo, el autor no tuvo en cuenta que Bestor sólo compensaba este poder dióptrico en personas con visión binocular normal y sin alteración de la acomodación y la convergencia. Por tanto, no se podría afirmar que se cumpla la misma 


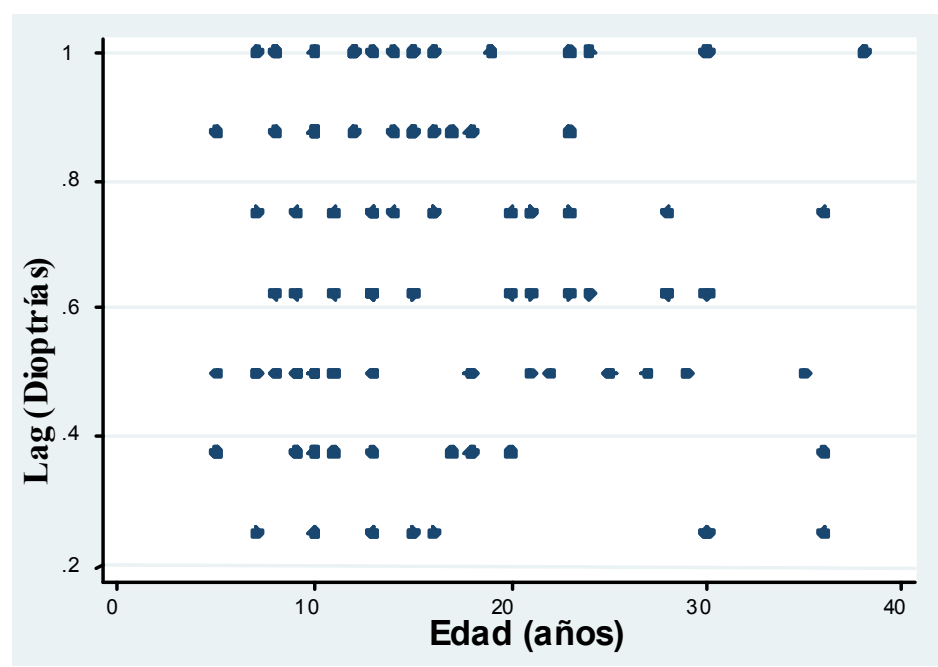

Gráfica 1. Relación entre el lag de acomodación y la edad

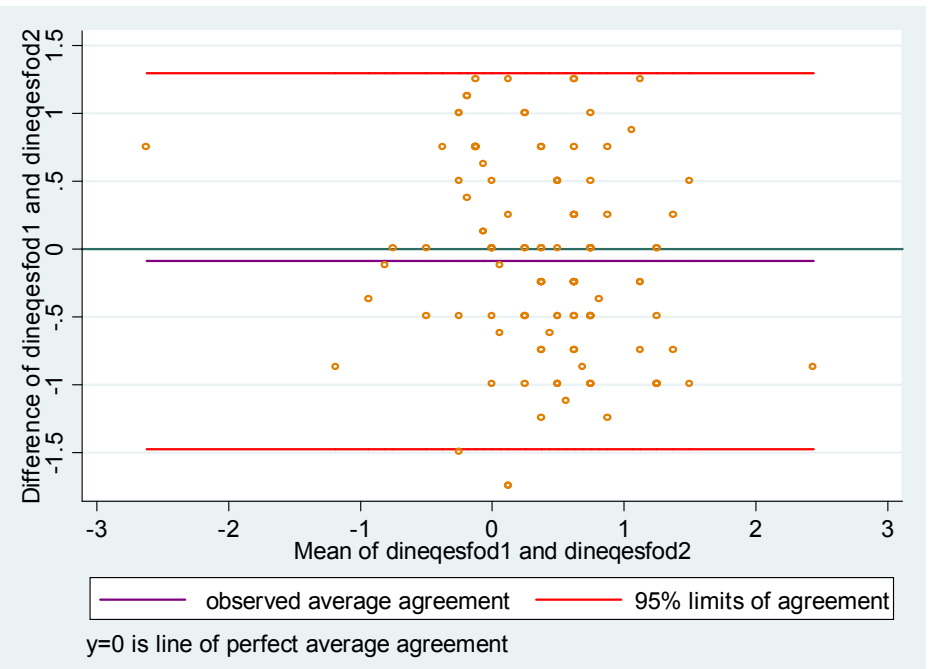

Grafica 2. Límites de acuerdo del $95 \%$ de Bland \& Altman retinoscopia dinámica monocular de Merchán ojo derecho

compensación en personas con alteración de la visión binocular como aún se aplica en la práctica clínica y sin evaluar previamente la respuesta acomodativa o lag de acomodación.

En cuanto a la reproducibilidad de las pruebas retinoscópicas, al determinar el poder refractivo en visión próxima con la retinoscopia de Nott se observó que presentó excelente reproducibilidad, mejor que la obtenida con MEM y entre estas dos pruebas, la reproducibilidad también fue excelente. Los resultados de esta investigación se relacionan con los estudios de Cacho ${ }^{8}$ y García ${ }^{9}$ quienes encontraron alta correlación entre MEM y Nott a pesar de determinar sólo la linealidad de los valores y no estimar su reproducibilidad. Asimismo, se confirma que Nott podría obtener una mayor reproducibilidad ${ }^{22}$ que MEM debido a que no se contamina la acomodación con la introducción de lentes en la línea visual, mientras que MEM u otros métodos podrían presentar resultados alterados por esta situación, según lo informan otros estudios ${ }^{7-9}$.

Sin embargo, no sólo la reproducibilidad entre examinadores o pruebas determina su calidad y por esta razón se evaluó el nivel de acuerdo con la prueba de los límites de acuerdo al 95\% de Bland \& Altman ${ }^{23}$, prueba aplicada con poca frecuencia en los estudios de evaluación de pruebas diagnósticas realizados en ciencias de la visión.

Entre estas dos pruebas, MEM y Nott el acuerdo correspondió a un valor aceptado clínicamente $( \pm 0,25 \mathrm{D})$, no hay diferencias significativas ${ }^{10}$, y por tanto presentan poca variabilidad. Con estos hallazgos se evidencia que los métodos son intercambiables para determinar el poder dióptrico en visión próxima, y estimar el valor de la respuesta acomodativa en personas menores de 40 años, aunque se sugeriría aplicar mejor el método de Nott por las consideraciones antes mencionadas (Gráficas 2 a 4).

En visión lejana se encontró que existe una excelente reproducibilidad ${ }^{12}$ entre los evaluadores al realizar la retinoscopia estática binocular en el ojo derecho $\mathrm{CCI}=0.89$, y en el ojo izquierdo $\mathrm{CCI}=0.80$ mientras que en la retinoscopia dinámica monocular se obtuvo una baja reproducibilidad CCI $=0.49$ en ambos ojos. Es posible que a pesar de controlarse la actividad del cristalino en la RDMM y se fije un punto próximo, influya en los resultados la hipermetropización de las personas, los cuales activan la acomodación $\mathrm{y}$, a su vez, variar el poder refractivo del ojo.

El límite de acuerdo entre los evaluadores evidencia variabilidad en todas las pruebas, siendo mayor en la RDMM $( \pm 1.50 \mathrm{D})$, mientras que en la retinoscopia estática binoculary monocular, $0.50 \mathrm{D}$ y $0.75 \mathrm{D}$ respectivamente.

Al determinar la concordancia en el estado refractivo, se observó que existe gran variabilidad entre los evaluadores así como también en el 


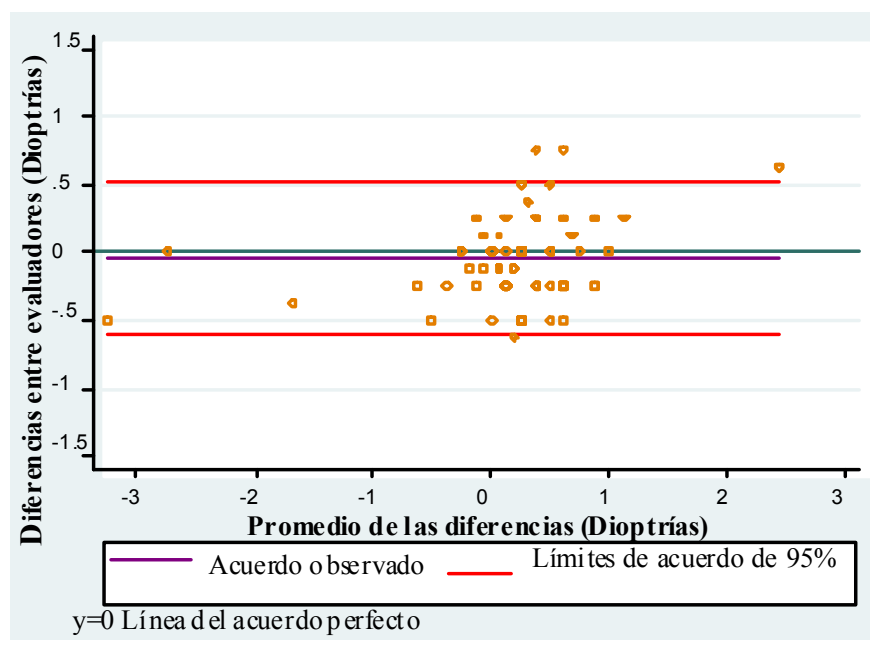

Grafica 3. Límites de acuerdo de $95 \%$ de Bland \& Altman retinoscopia estática ojo derecho

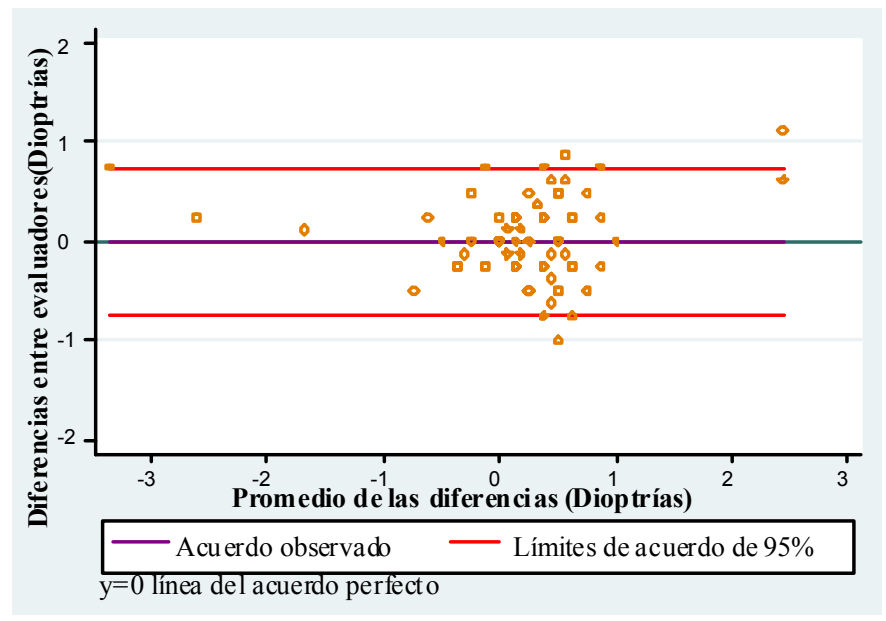

Gráfica 4. Límites de acuerdo de $95 \%$ de Bland \& Altman retinoscopia estática monocular ojo derecho

tipo de ametropía, cuando se evalúa con la retinoscopia dinámica monocular (22\%) esto quizás debido a que no controla la acomodación suficientemente y se genere alteración en el diagnóstico aún más cuando son defectos con poderes dióptricos de esfera y cilindro bajos, lo cual contradice la afirmación del autor en la que se refiere a que su técnica supera la retinoscopia estática binocular.

Por otro lado, la reproducibilidad entre la retinoscopia dinámica monocular de Merchán la estática, y la estática monocular, es moderada. El acuerdo entre esta prueba con la retinoscopia estática, corresponde a $\pm 1 \mathrm{D}$ y con la retinoscopia estática monocular de $\pm 0.75 \mathrm{D}$. Esto indica que la retinoscopia dinámica monocular no es una prueba reproducible ni presenta un acuerdo adecuado para determinar el poder dióptrico en visión lejana. Igualmente se afirma, que no presenta concordancia con estas pruebas de refracción para determinar ametropías o el estado emétrope en visión lejana. Lo contrario se demostró al comparar la retinoscopia estática binocular y monocular, las cuales presentaron una excelente concordancia $(\mathrm{CCI}>0.80)$ y el acuerdo fue $\pm 0.35 \mathrm{D}$ es decir $\pm 0,25 \mathrm{D}$, aproximándolo al cuarto de dioptría más cercano. Asimismo, la concordancia entre las pruebas para determinar el tipo de ametropía fue buena, (aproximadamente $70 \%)$.

Según estos hallazgos, se confirma la poca variabilidad entre estos métodos y se consideran intercambiables para los propósitos clínicos mencionados. Adicionalmente la hipercorrección negativa que se presentó en las retinoscopias estáticas (binocular y monocular) fue menor entre ellas (0.02D) que la obtenida al compararlas con la dinámica monocular $(0.18 \mathrm{D})$ aunque fueron valores clínicamente no significativos.

Con esta investigación se demuestra la confiabilidad de las pruebas retinoscópicas aplicadas en la práctica clínica para determinar el poder dióptrico y el estado refractivo en visión lejana y en visión próxima. Se confirma que la retinoscopia estática monocular, realizada en otros países para determinar el poder y estado refractivo en visión lejana es una prueba reproducible y de gran utilidad clínica, en personas con visión binocular normal y posiblemente en quienes presenten dificultad para el manejo del paciente $o$ alteraciones de la visión binocular.

Finalmente, los resultados de esta investigación evidencian científicamente que la calidad en términos de reproducibilidad de la retinoscopia dinámica monocular de Merchán, frecuentemente aplicada en Colombia y desconocida en el ámbito mundial, no es buena; por tanto, no podría considerarse dentro de la batería de pruebas aplicadas para determinar diagnósticos y correcciones refractivas tanto en visión lejana como en visión próxima, pues se sabe que una prueba para ser válida debe ser necesariamente reproducible.

Debido al diseño del estudio y métodos de recolección de la información los hallazgos se limitan a la población entre 5 y 39 años de edad, y a pesar de que 
el muestreo fue por conveniencia no se afectó la validez interna del estudio. Se sugiere realizar este tipo de estudio en personas con problemas de vergencias tipo tropia, no consideradas en esta investigación.

Conflicto de intereses. Los autores declaran que no hay conflicto de intereses en el presente manuscrito.

\section{REFERENCIAS}

1. Merchán de Mendoza G. Diez puntos sobre retinoscopia monocular. Optometria. 1966; 4: 33-8.

2. Borish I. Dynamic skyametry. In: Borish IM (ed.) Clinical refraction. Chicago: Professional Press; 1970.

3. Hodis S, Wood IC. Comparison of the techniques of videorefraction and static retinoscopy in the measurement of refractive error in infants. Ophthalmic Physiol Opt. 1994; 14 : 20-4.

4. Keith E, Lleywellyn R. Optometría. Retinoscopía. Barcelona: Ediciones Científicas y Técnicas SA; 1993. p. 92-6.

5. Rouse M, London R, Allen D. An evaluation of the monocular estimate method of dynamic retinoscopy. Am JOptom Physiol Optics. 1982; 59: 235-9.

6. Locke L, Somers W. A comparison study of dynamic retinoscopy techniques. Optom Vis Sci. 1989; 66: 540-4.

7. Rosenfield M, Portello JK. Comparison of clinical techniques to asses de near accommodative response. Optom Vis Sci. 1996; 73: 382-8

8. Cacho P, García A, García JR, López A. Comparison between MEM and NOTT dynamic retinoscopy. Optom Vis Sci .1999; 76: $650-5$.

9. García A, Cacho P. MEM and Nott dinamyc retinoscopy in patients with disorders of vergence and accommodation. Opthal Physiol Opt. 2002; 22: 214-20.
10. Goss D, Grosvenor T. Reliability of refraction a literatura review. J Am Optom Assoc. 1996; 67: 619-30.

11. Gilchrist J. QROC curves and kappa functions: New methods for evaluating the quality of clinical decisions. Opthal Physiol Opt. 1992; 2: 350-60.

12. Kraemer H. Evaluating medical test. Newbury Park: Sage publications; 1992.

13. American Optometric Association. Optometric clinical Guideline Practice. Care of the patient with accommodative and vergence disfunction. St. Louis: American Optometric Association; 1998.

14. Acuña LA. Validez y reproducibilidad de los métodos para diagnóstico y corrección de presbicia en personas entre 40 y 65 años de edad. Tesis de Maestría, Universidad Industrial de Santander, Bucaramanga; 2006.

15. Scheiman MM, Wick B. Tratamiento clínico de la visión binocular. Philadelphia: JB Lippincott Co; 1996.

16. Kramer H. How many subjects? California: Sage publications; 1.993

17. National Research council. [sede web] Acuity visual. New York. [Acceso: Junio de 2004]. Disponible en:www.aoa.org.com

18. CDC. Epilnfo, versión 6.04d. Epidemiología en ordenadores. Atlanta: CDC; 2001.

19. StataCorp. 2003. Stata Statistical Sofware: Release 9.0. College Station. Texas: Stata Corporation.

20. Wale R. Refractive errores and presbyopia. In: Jhonson G, Miassian D, Weale R, West S. (eds.).The epidemiology of eye disease. $2^{\mathrm{a}}$ ed. London: Arnold; 2003. p. 137-54.

21. Grossvenor T. Epidemiology or ametropia. In: Primary care optometry. New York: Butterworth; 2002. p.41-54.

22. Antona B, Sánchez I, Barrio A, Barra F, González E. Intraexaminer repeatability and agreement in accommodative response measurements. Opthalmic Physiol Opt. 2009; 29: 606-14.

23. Bland M, Altman D. Statistical methods for assessing agreement between two methods of clinical measurement. Lancet. 1986; 1: 307-10. 